Copyright C1997, American Institute of Aeronautics and Astronautics, Inc.

AIAA Meeting Papers on Disc, January 1997

A9715881, NAG3-1310, AIAA Paper 97-0885

\title{
Buoyancy and heater surface length effects in forced convection nucleate boiling
}

\author{
Herman Merte, Jr. \\ Michigan Univ., Ann Arbor
}

Steven U. Nestel

Emerson Electric Co., Ogden, UT

\begin{abstract}
AIAA, Aerospace Sciences Meeting \& Exhibit, 35th, Reno, NV, Jan. 6-9, 1997
Subcooled forced convection nucleate boiling experiments were conducted with R113 using thin gold film on quartz and gold-oated copper substrate flat heaters, as preliminary research for future studies in microgravity. Orientation relative to gravity, flow velocity, liquid subcooling, heat flux, and heater length aspect ratio were varied to provide a better understanding of the relative roles of various heater transfer mechanisms. Microlayer evaporation is expected to play a critical role in microgravity. Increasing the orientation angles from horizontal up toward the downward facing orientations results in an enhancement in the heat transfer with the gold film heater, as does increasing the length of the heated surface. A flow velocity increase generally decreases the overall heat transfer, except for the cases where dryout occurs as the lower velocities. The enhancement of the boiling process taking place with low velocities for those orientations is attributed to 'sliding' vapor bubbles, which result in the continuous renewal of the thin liquid layers beneath the bubbles. (Author)
\end{abstract}




\title{
Buoyancy and Heater Surface Length Effects in Forced Convection Nucleate Boiling"
}

\author{
Herman Merte, Jr. \\ The University of Michigan \\ Department of Mechanical Engineering \\ and Applied Mechanics \\ Ann Arbor, MI 48109-2125 \\ Steven U. Nestel \\ Emerson Electric Company \\ Ogden, UT 84404
}

\begin{abstract}
Subcooled forced convection nucleate boiling experiments were conducted with R 113 using thin gold film on quartz and gold coated copper substrate flat heaters, as preliminary research for future studies in the microgravity of space. Orientation relative to gravity, flow velocity, liquid subcooling, heat flux, and heater length aspect ratio were varied to provide a better understanding of the relative roles of various heater transfer mechanisms. Under microgravity conditions, in the absence of buoyancy, vapor bubbles are expected to remain adhered to or near the heater surface, motivating the choice of the downward facing orientations in the present work. Shown to account for a majority of the total heat transfer of these conditions, microlayer evaporation is expected to play a critical role in microgravity. Increasing the orientation angles from horizontal up toward the downward facing orientations results in an enhancement in the heat transfer with the gold film heater, as does increasing the length of the heated surface. A flow velocity increase generally decreases the overall heat transfer, except for the cases where dryout occurs as the lower velocities. The enhancement of the boiling process taking place with low velocities for those orientations is attributed to "sliding" vapor bubbles, which result in the continuous renewal of the thin liquid layers beneath the bubbles.
\end{abstract}

\section{Introduction}

Boiling is a rather imprecise term applied to the process of evaporation in which the rate of liquidvapor phase change is large. In seeking to determine the role and significance of body forces on the process, of which buoyancy or gravity is just one agent, it becomes necessary to define boiling more precisely. It is generally characterized by the formation and growth of individual vapor bubbles arising from heat transfer to the liquid, either at a solid/liquid or liquid/liquid interface, or volumetrically. For present purposes the superheating of the liquid required will be considered to take place only at solid surfaces. The terms "bubble" boiling and "nucleate" boiling are frequently used, in recognition of the interactions of surface tension and other forces in producing discrete bubbles at distinctive locations (although not always). These discrete bubbles may be small or large, depending on the nature of external forces acting (e.g. - high velocity forced convection boiling for the former, and pool boiling in microgravity for the latter).

It has become clear that the enhancement of the heat transfer with boiling is a consequence of the rapid evaporation of a superheated liquid. This evaporation can only occur at an existing liquidvapor interface, the initial formation of which is termed nucleation. A requirement for sustaining the large heat transfer of boiling, as characterized by Cooper 1 , is that the "equivalent liquid path length" must be quite small, to which must be added, "and capable of rapid replenishment". Whatever the combination of circumstances of heater surface geometry, bulk liquid velocity, body forces, and liquid temperature distribution, both superheated and subcooled, that contribute to the formation and renewal of such thin liquid layers following their evaporation, it can be anticipated that enhancement of the heat transfer will follow. This implies also that the vapor formed must be removed from the vicinity of the heater surface, which otherwise would result in dryout, or the Critical Heat Flux.

The concept of a liquid microlayer forming and evaporating beneath growing vapor bubbles was given a solid foundation based on measurements in the work of Moore and Mesler 2 , Cooper and Lloyd 3 , Jawurek ${ }^{4}$, and Kenning and Yan 5 , all with pool boiling. Measurements with long term pool boiling 
in microgravity 6 have demonstrated that the heat transfer is enhanced considerably over that in earth gravity at the lower levels of heat flux, but that the heat flux at which dryout takes place is substantially reduced. The enhancement is attributed to the steady existence of a liquid "macrolayer" between the heater surface and a large vapor bubble which remains suspended in the vicinity of the heater. This layer serves as a boundary across which evaporation takes place, as well as a mechanism for the efficient removal of vapor bubbles from the heater surface, due to vapor pressure differences arising from surface tension.

It may be expected that any bulk liquid motion imposed parallel to the heater surface in microgravity would further enhance the heat transfer process if what has been termed sliding bubbles are present, in a manner similar to that produced by buoyancy in earth gravity 7 and by forced flow in short term reduced gravity ${ }^{8}$. These result in the continuous renewal of the thin liquid layers beneath the bubbles. The departure size of the vapor bubble is an important constituent in the microlayer evaporation process taking place in such circumstances, and the drag forces in forced convection boiling provide a vehicle for this departure in microgravity. A description of the relation between the buoyancy dominant and inertial dominant regimes for bubble departure has been provided in terms of a "two-phase Richardson number" 9 .

The elements that constitute the nucleate boiling process - nucleation, growth, motion, and collapse of the vapor bubbles (if bulk liquid is subcooled) - are common to both pool and flow boiling. It is well known that the imposition of bulk liquid motion affects the vapor bubble behavior relative to pool boiling, but does not appear to significantly influence the heat transfer. Indeed, it has been recommended in the past that empirical correlations or experimental data of pool boiling be used for design purposes with forced convection nucleate boiling 10,11. It is anticipated that such will most certainly not be the case for boiling in microgravity, based on preliminary observations to be described below. In earth gravity buoyancy will act to remove the vapor bubbles from the vicinity of the heater surface regardless of how much the imposed bulk velocity is reduced, depending, of course, on the geometry of the system. The major socalled forces governing the motion of the bubbles are buoyancy, liquid momentum and viscosity. With sufficiently high flow Reynold's Numbers, it can be intuited that the latter two forces will outweigh the first, and the process will be the same whether at earth gravity or microgravity. However, as the
Reynold's Number is reduced the magnitude of the liquid momentum and viscous forces are correspondingly reduced, and in microgravity buoyancy cannot take over as a "back-up" mechanism for vapor removal, leaving only the reduced levels of liquid momentum and viscous forces. Vapor bubbles have been observed to dramatically increase in size in the limit of pool boiling in microgravity 12 , but will be bounded in size by the action of the liquid momentum and viscous forces of forced convection in microgravity.

Certain other effects that can be neglected at normal earth gravity, such as surface tension, both at the solid-liquid-vapor contact line and at the liquidvapor surface associated with the interface temperature variation, will be of consequence at microgravity conditions. The net quantitative effect of these on the vapor bubble behavior is unknown, at present, as are the related effects on the heat transfer, and provides one of the motivations for the study of the flow boiling process in microgravity. As a case in point, the departure size of vapor bubbles at low velocity in microgravity will be an important parameter in the design of vaporizing heat exchangers for space applications, since its size relative to that of the flow passage has a significant influence on the mechanisms and magnitudes of the local heat flux.

The objective of the present work is to provide measurements further demonstrating that the enhancement of boiling heat transfer with forced convection is a consequence of the evaporation of the thin films formed beneath the growing vapor bubbles. The measurements also provide qualitative indications of the trends in heat transfer to be expected with flow boiling in microgravity. The procedure used is to vary the orientation between a flat heater surface and gravity, as well as the length of the heated surface in the flow direction, parallel to the heater. A flat heater surface is selected in order to provide a well defined and consistent orientation between the heater surface, the flow direction and earth gravity.

\section{Experimental Apparatus}

A schematic of a low velocity forced convection boiling loop for proposed studies in microgravity is shown in Figure 1. Velocities can be varied from $0.5 \mathrm{~cm} / \mathrm{s}$ to $60 \mathrm{~cm} / \mathrm{s}$ by pump speed control combined with changes in test section height, from $2.54 \mathrm{~cm}$ (1 inch) to $0.318 \mathrm{~cm}(0.125 \mathrm{inch})$. The use of a flow loop permits the study of boiling under steady conditions as well as under transients, and thus can accommodate the use of metallic surfaces which, while representative of engineering surfaces, also introduce complications with transients, 


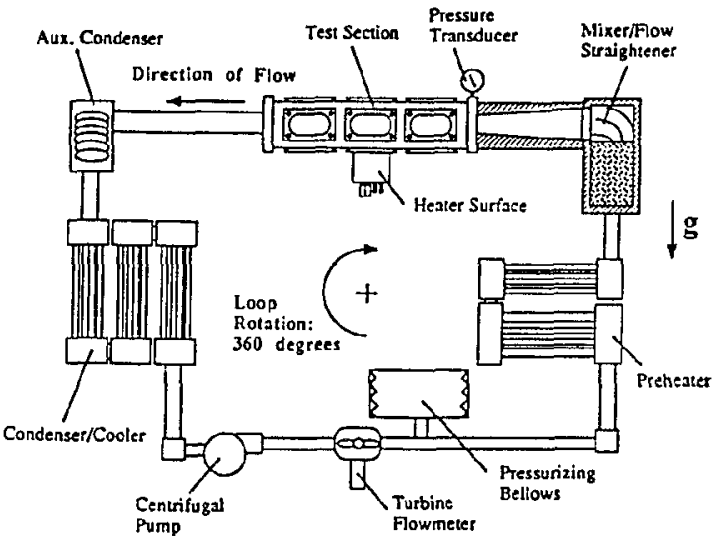

Figure 1. Forced convection boiling loop.

associated with heat capacity effects. The flow loop proper occupies a volume of about $1.22 \times .61 \times .46 \mathrm{~m}$ $(48 \times 24 \times 18$ inches). The flat heater surfaces used are rectangular in shape, $1.91 \mathrm{~cm}$ by $3.81 \mathrm{~cm}(0.75 \mathrm{x}$ 1.5 inches), consisting of a 400 Angstrom thick semitransparent gold film sputtered on a quartz substrate which serves simultaneously as a heater and a resistance thermometer, shown in Figure 2, or a gold plated copper substrate of the same size, Figure 3.

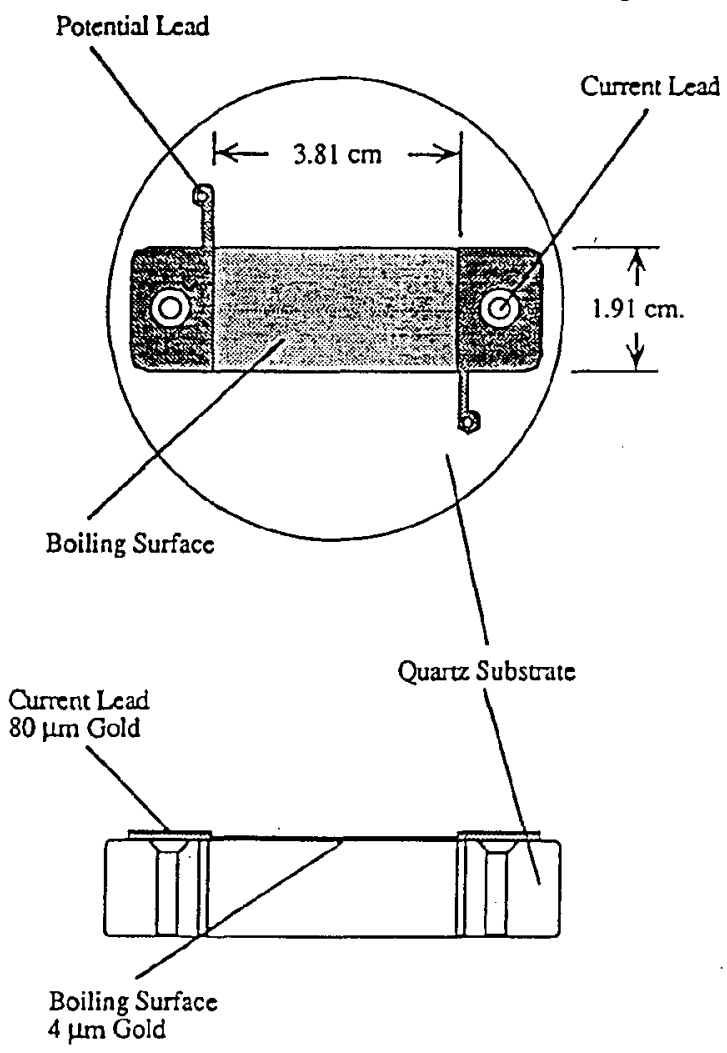

Figure 2. Thin film gold on quartz substrate heater.
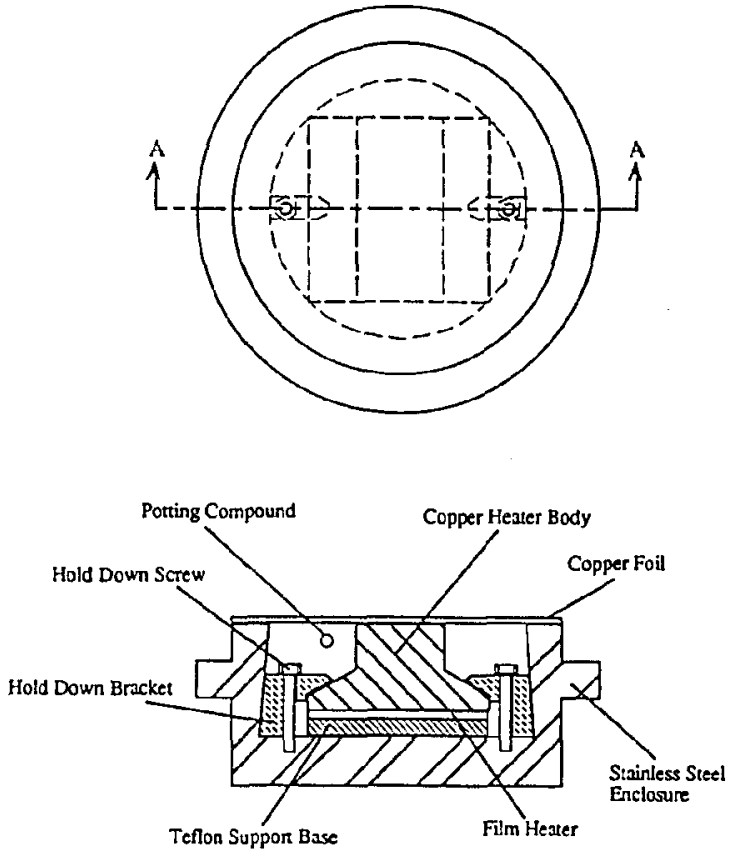

Section A-A

Figure 3. Gold coated copper substrate heater.

The heaters are mounted on a disc which can be rotated so that the heated length in the flow direction can be changed from 1.91 to $3.81 \mathrm{~cm}(0.75$ to 1.5 inches). Variable buoyancy normal to the heater surface is achieved by rotation of the entire loop relative to earth gravity. Of course, this is at the expense of varying the buoyancy parallel to the heater surface. The $0^{\circ}$ reference for orientation is taken with the flat heater surface horizontal facing upward, while the angle increases such that $90^{\circ}$ implies a vertical heater surface with the fluid flow direction upward.

The surface temperature of the copper substrate heater in Figure 3 is measured with a calibrated chromel-constantan thermocouple inserted in a $0.76 \mathrm{~mm}$ ( 0.030 inches $)$ diameter hole parallel to the surface, with its center $0.76 \mathrm{~mm}$ (0.030 inches) below the surface. A smooth copper foil $0.0254 \mathrm{~mm}$ ( 0.001 inches) thick is soldered to the copper heater body and to the stainless steel enclosure to eliminate the artificial boiling that otherwise would result at the heater edge.

Degassed R-113 was used as the working fluid, with degassing and purification taking place by a combination of distillation, filtration with a molecular sieve, and freezing on liquid nitrogen cooled surfaces while pumping off non-condensables. The adequacy of degassing was established by 
comparing the measured vapor pressure with published saturation values over the temperature ranges of interest, within the respective laboratory accuracies of $\pm 0.17 \mathrm{kpa}( \pm 0.025 \mathrm{psi})$ and $\pm 0.06^{\circ} \mathrm{C}$ $\left( \pm 0.1^{\circ} \mathrm{F}\right)$

A pressure control system actively maintains the pressure in the loop constant to within $\pm 0.7 \mathrm{kpa}( \pm 0.1 \mathrm{psi})$, with the bellows shown in Figure 1. The bulk fluid temperature at the inlet of the test section is maintained constant at a nominal value of $49^{\circ} \mathrm{C}$ to within $\pm 0.2^{\circ} \mathrm{C}$ for any test, while the flow rate was controlled to within $2-5 \%$ of the set value, depending on the flow rate. Bulk liquid subcooling was changed by varying the system pressure.

The uncertainty in the steady heat flux measurements for both heaters of Figures 2 and 3 was $\pm 0.25 \mathrm{w} / \mathrm{cm}^{2}$. The uncertainty in the measured mean surface temperature for the gold film heater of Figure 2 was $\pm 1{ }^{\circ} \mathrm{C}$, while that for the copper substrate heater of Figure 3 was $\pm 0.1^{\circ} \mathrm{C}$. Further details are available in the work of Nestel and Merte ${ }^{13}$.

\section{Experimental Procedures}

The emphasis in the results to be presented below is concerned with the influence of the heated length in the flow direction on the heat transfer. However, as will become obvious, this effect cannot be considered separate from the other independent variables involved in the flow boiling process currently restricted to earth gravity: mean heater surface heat flux, heater surface orientation relative

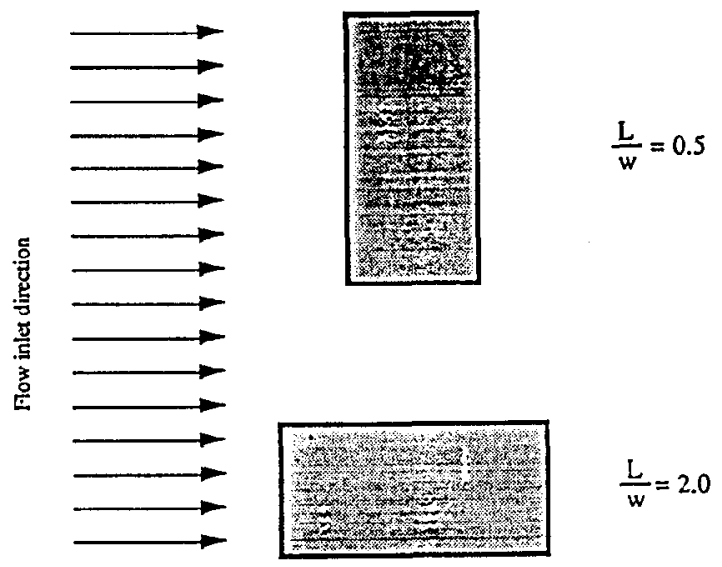

Figure 4. Heater surface length orientation used for flow parallel to heater. to earth gravity, and bulk liquid velocity and subcooling, defined at the test section entrance for consistency. The enhancement of the boiling process taking place with low velocities alone for those orientations producing "sliding" vapor bubbles has been demonstrated and presented previously 14, 15, and will not be repeated here.

The measurements will be presented such that the effect of the heated length over an appropriate range of heat flux can be observed simultaneously with the effect of another independent parameter, either bulk liquid subcooling, velocity, or system orientation. Figure 4 shows the symbols used to indicate the heated length in each of the figures following.

All results presented were obtained under steady operating conditions, with step increases in heat flux followed by sufficient time to reach a new steady state prior to recording the data. Dryout of the heater occurred for several test conditions, requiring termination of testing. Also, certain combinations of orientation and subcooling could not be used because of cavitation in the pump.

\section{Results}

Figure 5 compares the established boiling effectiveness between the two lengths used for the two heater surfaces for identical conditions of high velocity $(\mathrm{V}=32.4 \mathrm{~cm} / \mathrm{s})$, the highest subcooling $(\Delta \mathrm{T}$ sub $=11.1^{\circ} \mathrm{C}$ ) and the heater surface facing horizontal down $\left(\theta=180^{\circ}\right)$. For a given level of the heat flux, the heater surface superheat represents a measure of the effectiveness of the heat transfer, with effectiveness increasing as the heater surface superheat decreases. It is noted that the heated length has relatively small influence with this high velocity

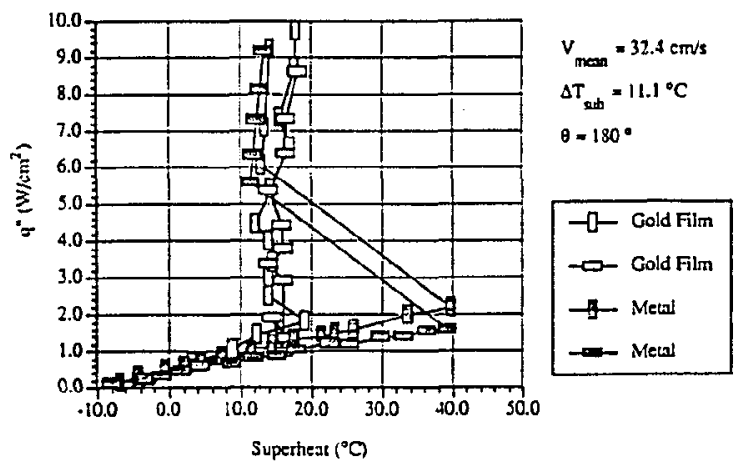

Figure 5. Comparison of gold film on quartz with copper substrate heater, for high velocity $(32.4 \mathrm{~cm} / \mathrm{s})$ and subcooling $\left(11.1^{\circ} \mathrm{C}\right)$, horizontal down orientation $\left(\theta=180^{\circ}\right)$. 
and subcooling, as manifested by the small differences in heater surface superheat. Once nucleation takes place the metal substrate becomes a more effective heater surface because of the presence of more nucleating sites compared to the polished quartz substrate, as is well known. The data in the lower part of Figure 5 represent the single phase heat transfer results, in which it is seen that the heat flux is reduced (for a given heater surface superheat) with the longer heater, due to the increased boundary layer temperature level. The single phase results will be omitted in all of the subsequent results for purposes of clarity. The fact that the heater surface superheat prior to nucleation was considerably higher with the metal substrate than with the polished quartz substrate is consistent with other measurements in flow boiling 15 and with corresponding measurements in pool boiling 16 , and is attributed to the high degree of wettability associated with $R-113$.

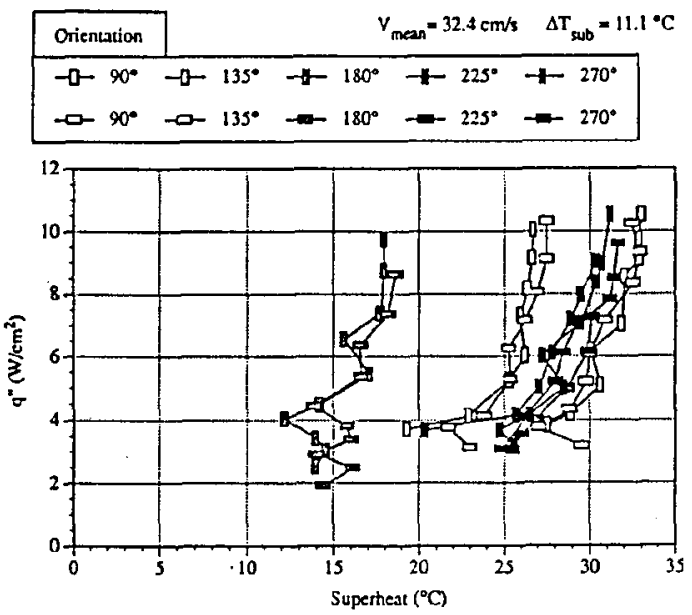

Figure 6. Quartz substrate heater at high velocity $(32.4 \mathrm{~cm} / \mathrm{s})$ and subcooling $\left(11.1^{\circ} \mathrm{C}\right)$ with various orientations.

Measurements with the gold film on quartz substrate heater alone are given in Figure 6 for the largest velocity and subcooling used, for a variety of orientations. The difference in behavior with heater length is not manifested at this high velocity and subcooling, including the horizontal upward facing orientation $\left(\theta=0^{\circ}\right)$, omitted for clarity. As the surface is rotated from vertical upflow, $\theta=90^{\circ}$, to the horizontal down position, $\theta=180^{\circ}$, enhancement in the heat flux occurs for all levels of heat flux. At the orientations of $225^{\circ}$ and $270^{\circ}$, the heat transfer diminishes due to the formation of dry patches on the heater surface, associated with the tangential component of buoyancy acting against the flow drag force. The data at $180^{\circ}$ is identical to that shown for the quartz substrate in Figure 5, with the abscissa expanded.

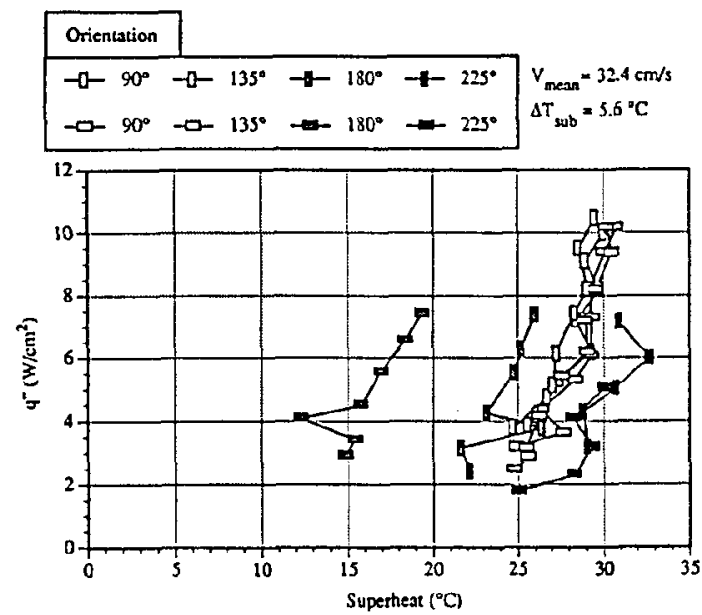

Figure 7. Quartz substrate heater at high velocity $(32.4 \mathrm{~cm} / \mathrm{s})$ and medium subcooling $\left(5.6^{\circ} \mathrm{C}\right)$ with various orientations.

The experimental conditions represented in Figure 7 are identical to that for Figure 6 , except that a medium subcooling level of $5.6^{\circ} \mathrm{C}$ is used, instead of the $11.1{ }^{\circ} \mathrm{C}$ in Figure 6 . The reduction in subcooling is accompanied by a general degradation in the heat transfer, except for the longer heater in the horizontal downward orientation $\left(\theta=180^{\circ}\right)$, which remains virtually unchanged. This is attributed to the action of buoyancy in maintaining an effective evaporating thin liquid film independent of the subcooling level.

Figure 8 and 9 apply to experimental conditions similar to that in Figure 7 , with the medium subcooling of $5.6{ }^{\circ} \mathrm{C}$, except that the bulk fluid velocity is reduced from $32.4 \mathrm{~cm} / \mathrm{s}$ to $18.1 \mathrm{~cm} / \mathrm{s}$ and $12 \mathrm{~cm} / \mathrm{s}$, respectively, and only orientations of $\theta$ $=135^{\circ}$ and $\theta=180^{\circ}$ are given. The formation of dry patches with $\theta=270^{\circ}$ terminated the experiments for

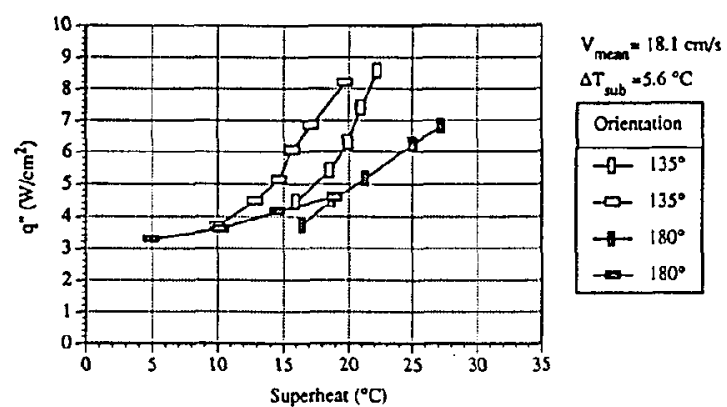

Figure 8. Quartz substrate heater at medium velocity $(18.1 \mathrm{~cm} / \mathrm{s})$ and medium subcooling $\left(5.6^{\circ} \mathrm{C}\right)$ with orientations of $\theta=135^{\circ}, 180^{\circ}$. 


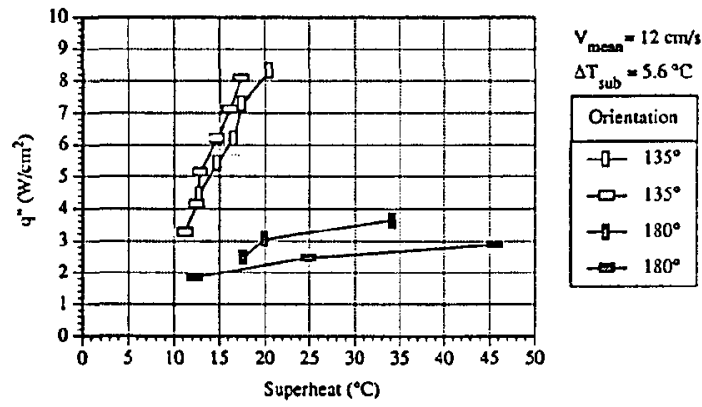

Figure 9. Quartz substrate heater at low velocity $(12 \mathrm{~cm} / \mathrm{s})$ and medium subcooling $\left(5.6^{\circ} \mathrm{C}\right)$ with orientations of $\theta=135^{\circ}, 180^{\circ}$.

the lower subcoolings of $5.6^{\circ} \mathrm{C}$ and $2.8^{\circ} \mathrm{C}$. On comparing Figures 7 and 8 it is noted that for the same subcooling and for both orientation angles $\theta=$ $135^{\circ}$ and $\theta=180^{\circ}$, that reducing the bulk liquid velocity results in an enhancement of the heat transfer. This is a consequence of the increasing net vapor production accompanying the lower velocity with the reduced subcooling, which enhances the thin liquid film evaporation associated with these orientations. This enhanced behavior with velocity reduction is also observed in Figure 9 , but only for the orientation angle $\theta=135^{\circ}$. Dryout takes place at $\theta=180^{\circ}$ with low levels of heat flux for the low velocity of $12 \mathrm{~cm} / \mathrm{s}$, accompanied by significant increases in heater surface superheat.

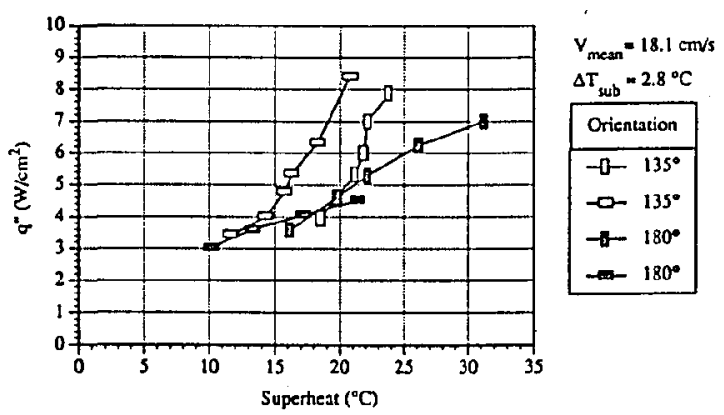

Figure 10. Quartz substrate heater at medium velocity $(18.1 \mathrm{~cm} / \mathrm{s})$ and low subcooling $\left(2.8^{\circ} \mathrm{C}\right)$ with orientations of $\theta=135^{\circ}, 180^{\circ}$.

Figure 10 applies to the identical experimental conditions of Figure 8 , with the medium velocity of $18.1 \mathrm{~cm} / \mathrm{s}$, except that the bulk liquid subcooling is reduced from $5.6^{\circ} \mathrm{C}$ to $2.8^{\circ} \mathrm{C}$, almost saturated. The tendency for early dryout, limiting the maximum heat flux, is noted for the longer heater surface in the horizontal down orientation $\left(\theta=180^{\circ}\right)$, as is also the case in Figure 8 . On the other hand, to be noted in both Figures 8 and 10 , the heat transfer is enhanced for the longer heater surface with $\theta=135^{\circ}$, where the buoyancy of the additional vapor formed serves to increase the vapor velocity parallel to the heater surface.

The influence of the heater length in the flow direction on the heat transfer is quite different with the copper substrate heater from that with the quartz substrate. The copper substrate tends to provide a more uniform temperature in the flow direction, with a widely varying local heat flux, whereas the thin gold film heater on the quartz substrate tends to provide a more uniform heat flux in the flow direction, with a varying local temperature. The heated length with the copper substrate appears to influence the heat transfer only at the highest velocity used here. The behavior with this velocity $(32.4 \mathrm{~cm} / \mathrm{s})$ and the highest subcooling level $(11.1$ $\left.{ }^{\circ} \mathrm{C}\right)$ for the horizontal down orientation $\left(\theta=180^{\circ}\right)$ was presented previously in Figure 5. Measurements at this same velocity but for the medium subcooling $\left(5.6^{\circ} \mathrm{C}\right)$ are given in Figure 11 for orientations of $\theta=$ $0^{\circ}, 135^{\circ}, 180^{\circ}$. For the horizontal up orientation $(\theta=$ $0^{\circ}$ ), increasing the heated length degrades the heat transfer, whereas no effect of the heated length was observed with the quartz substrate heater. Also to be contrasted with the quartz substrate heater in Figure 7 , the horizontal down orientation $\left(\theta=180^{\circ}\right)$ with the copper substrate heater in Figure 11 results in a degradation of the heat transfer relative to the other orientations. However, the longer heater remains more effective. Figure 12 provides a direct comparison of the influence of the heated length at
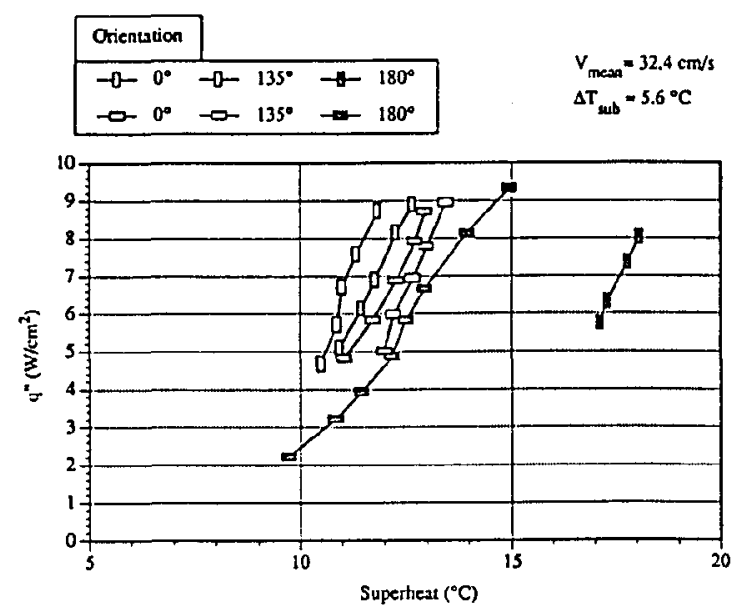

Figure 11. Copper substrate heater at high velocity $(32.4 \mathrm{~cm} / \mathrm{s})$ and medium subcooling $\left(5.6^{\circ} \mathrm{C}\right.$ ) with orientations of $\theta=0^{\circ}, 135^{\circ}, 180^{\circ}$. 


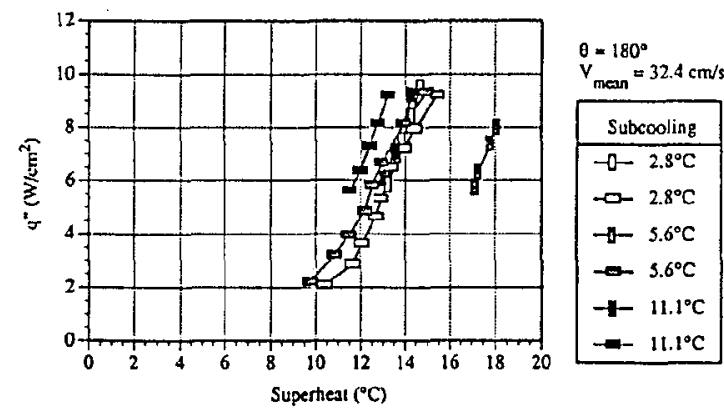

Figure 12. Copper substrate heater at high velocity $(32.4 \mathrm{~cm} / \mathrm{s})$ and horizontal down orientation. Direct comparison of influence of subcooling.

the various subcoolings, for the high velocity $(32.4$ $\mathrm{cm} / \mathrm{s})$ and horizontal down orientation $\left(\theta=180^{\circ}\right)$ case with the copper substrate heater of Figure 11. It is noted that as the subcooling level is increased, the shorter heater experiences first a decrease and then a return to the original effectiveness, indicating the presence of competing mechanisms in the net heat transfer process. This is attributed to changes in the net vapor volume generated over this heated length. At bulk fluid velocities lower than that in Figures 11 and 12 and for all subcooling levels used here, changing the heater length had little or no influence on the heat transfer. Details of these cases are available in Nestel and Merte 13.

Figure 13 presents a direct comparison, for the quartz substrate heater, of the influence of the heated length at the various flow velocities, for the high subcooling level $\left(11.1^{\circ} \mathrm{C}\right)$ and horizontal down orientation $\left(\theta=180^{\circ}\right)$. No effect of length is noted at the highest velocity, while a "crossover" occurs at the medium velocity with some degradation, and early dryout takes place at the lowest velocity. The crossover to increasing degradation at the medium

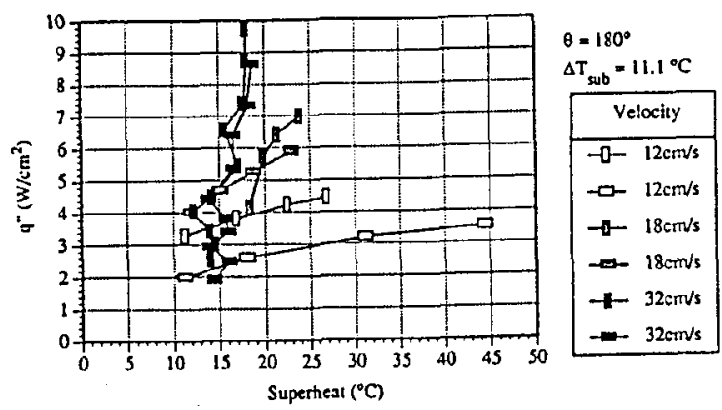

Figure 13. Quartz substrate heater at high subcooling $\left(11.1^{\circ} \mathrm{C}\right)$ and horizontal down orientation $\left(\theta=180^{\circ}\right)$. Direct comparison of influence of flow velocity. velocity takes place as the heat flux is increased, and is attributed to the larger vapor generation rate per unit heater width with increasing length, which interferes with the flow of liquid to the heater surface.

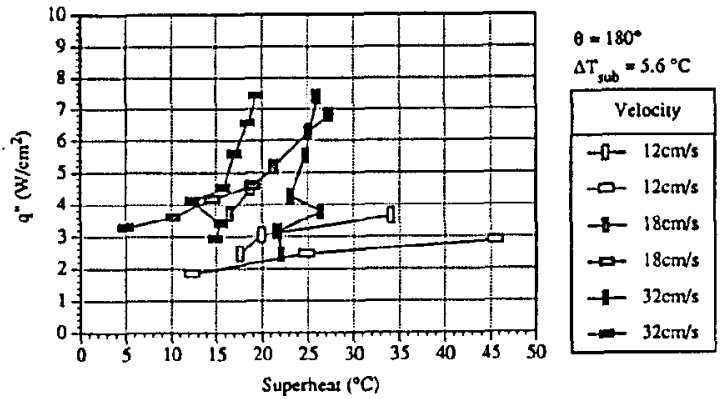

Figure 14. Quartz substrate heater at medium subcooling $\left(5.6^{\circ} \mathrm{C}\right)$ and horizontal down orientation $\left(\theta=180^{\circ}\right)$. Direct comparison of influence of flow velocity.

Figure 14 is similar to Figure 13, except that the subcooling level is decreased to $5.6^{\circ} \mathrm{C}$. The heater surface length now has an influence even at the highest velocity, with enhancement of the heat transfer for the longer surface. This effect is reversed for the lowest velocity, while a crossover tendency is noted at the intermediate velocity.

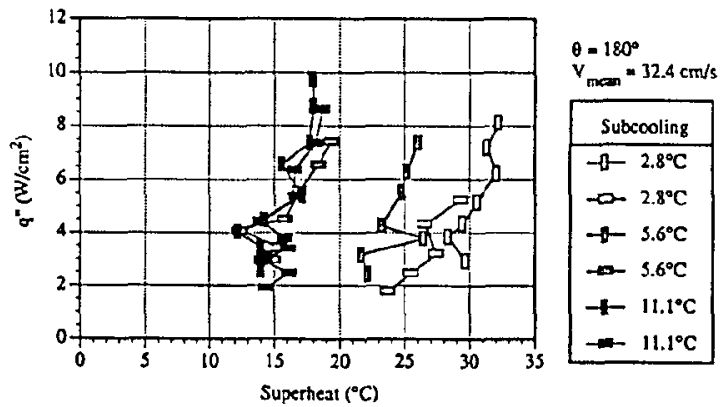

Figure 15. Quartz substrate heater at high velocity $(32.4 \mathrm{~cm} / \mathrm{s})$ and horizontal down orientation $\left(\theta=180^{\circ}\right.$ ). Direct comparison of influence of subcooling.

Figure 15 gives a direct comparison of the influence of the heated length at the various subcoolings, for the high velocity $(32.4 \mathrm{~cm} / \mathrm{s})$ and horizontal down orientation $\left(\theta=180^{\circ}\right)$ case with the quartz substrate heater of Figure 7 . The high subcooling case $\left(11.1^{\circ} \mathrm{C}\right)$ is identical to that in Figure 5 . As with the copper substrate heater in Figure 12, a 
maximum in the effect of the heater length occurs at the intermediate subcooling level $\left(5 \cdot 6^{\circ} \mathrm{C}\right)$. However, as is seen in Figure 15, a decrease in the subcooling level results in a degradation of the heat transfer process, manifested by the increase in heater surface superheat, in contrast to the behavior with the copper substrate in Figure 12, where the heater superheat level returns to that for the high subcooling case. This difference in behavior is believed to be a consequence of the difference in the nucleating characteristics between the metal and polished quartz substrate: The polished quartz surface requires a considerably greater superheat for nucleation, which is attained at the expense of less net vapor production, for a given heat flux level at the heater surface. Less vapor production results in a reduction of the evaporation of any thin liquid films between the heater surface and "sliding" vapor bubbles. At the intermediate subcooling level $\left(5.6^{\circ} \mathrm{C}\right)$ in Figure 15 the increased heater length is postulated to permit more effective liquid film evaporation than with the lower and higher subcooling levels. Confirmation of this behavior will require local heater surface temperature measurements in the flow direction.

\section{Conclusions}

Measurements of heater surface superheats are presented for subcooling forced convection nucleate boiling, using a thin gold film heater on a quartz substrate and a copper substrate heater, while varying the heater length in the flow direction, the orientation relation to gravity, the liquid flow velocity and subcooling, and the heat flux. For a given level of the heat flux, the heater surface superheat represents a measure of the effectiveness of the heat transfer process, with effectiveness increasing as the heater surface superheat decreases. The primary parameter of interest in the present study is the influence of the heated length in the flow direction on this heat transfer effectiveness.

While generalized quantitative conclusions are not yet possible, based on the results presented here a single qualitative inference can be drawn about the interactions among the parameters. Whatever combinations of these parameters contribute to the formation and renewal of a thin liquid layer between a solid heater surface and a vapor bubble will enhance the heat transfer process. For the flat heater surfaces used here, any orientation in which buoyancy serves to hold that the vapor bubble against the heater surface, while still permitting its motion and removal, will result in enhancement of the beat transfer. The heater surface nucleating characteristics, bulk liquid velocity and subcooling, and heat flux levels introduce elements whose effects depend on the resulting rate of vapor generation, motion and removal from the vicinity of the heater surface. Such a statement may, at first glance, appear to be almost banal. However, the ultimate objective of predictive capabilities, which provides a measure of the understanding of a process, will not be possible until the net effects of these various parameters are satisfactorily quantified.

\section{Acknowledgment}

The research presented here was conducted under NASA Grant NAG3 - 1310. The interest and support of Dr. Francis Chiaramonte and Mr. Jack Salzmann at the NASA Lewis Research Center are sincerely appreciated.

\section{References}

1. Cooper, M.G., "Effects of Orientation in Nucleate Pool Boiling", Collected Papers in Heat Transfer 1988 - Vol. 2, HTD - Vol. 104, presented at Winter Annual Meeting of ASME November 27 - December 2, 1988, pp $165-168$

2. Moore, F.D. and Mesler, R.B., "The Measurement of Rapid Surface Temperature Fluctuations During Nucleate Boiling of Water" AIChE Journal, Vol. 7, No. 4, 1961, pp 620-624.

3. Cooper, M.G. and Lloyd, A.J.P., "The Microlayer in Nucleate Pool Boiling" Int. Journal of Heat and Mass Transfer, Vol. 12. 1969, pp 895-913.

4. Jawurek, H.H., "Simultaneous Determination of Microlayer Geometry and Bubble Growth in Nucleate Boiling", Int. Journal of Heat and Mass Transfer, Vol. 12, 1969, pp 843-848.

5. Kenning, D.B.R. and Yan, Y., "Pool Boiling Heat Transfer on a Thin Plate: Features revealed by Liquid Crystal Thermography", Int. Journal of Heat and Mass Transfer, Vol. 39, No. 15, 1996, pp 3117-3137.

6. Lee, H.S., Merte, H., Jr., and Chiaramonte, F., "The Pool Boiling Curve in Microgravity", AIAA Paper 96-0499 presented at 10th Annual Symposium on Microgravity Science and Space Processing, AIAA 34th Aerospace Science Meeting, Jan. 15-18, 1996, Reno, NV. To be published in AIAA Journal of Thermophysics and Heat Transfer, 1997. 
7. Cornwell, K., "The Role of Sliding Bubbles in Boiling on Tube Bundles, " Heat Transfer 1990 - Vol. 3, Proc. 9th Int. Heat Transfer Conf., Jerusalem, Israel, Aug. 19-24, 1990, pp 455-460.

8. Cooper, M., et al, "Behavior of Vapour Bubbles Growing at a Wall with Forced Flow," Int. J. Heat Mass Transfer, 26, 1983, pp 1489-1507.

9. Kirk, K.M., and Merte, H., Jr., "A Mixed Natural/Forced Convection Nucleate Boiling Heat Transfer Criteria," Heat Transfer 1994, Vol. 7, pp 479-484. Proceedings of the 10th International Heat Transfer Conference, Brighton, England, August 14-18, 1994.

10. Bergles, A.E., and Rohsenow, W.M., "The Determination of Forced-Convection Surface Boiling Heat Transfer," J. Heat Transfer, 86, 1964, pp 365-382.

11. Bartolini, R. et al, "Experimental Study on Nucleate Boiling of Water in Vertical Upflow and Downflow," Int. J. Multiphase Flow, 9, 1983, pp 161-165.

12. Merte, H., Jr., Lee, H.S., and Keller, R.B., "Report on Pool Boiling Experiment Flown on STS-47 (PBE-IA), STS-57 (PBE-IB), and STS-60 (PBE-IC). NASA CR 198465 prepared for Lewis Research Center under Contact NAS3-25812. March, 1996.
13. Nestel, S.U., and Merte, H., Jr., "The Effects Heater Surface Length in Forced Convection Nucleate Boiling," Report No. UM-MEAM95-07, University of Michigan, Department of Mechanical Engineering and Applied Mechanics. Conducted under NASA Grant NAG3-1310, July 1995.

14. Merte, H., Jr., "Pool and Flow Boiling in Variable and Microgravity," 2nd Microgravity Fluid Physics Conference, June 21-23, 1994, NASA-Lewis, Cleveland, OH.

15. Kirk, K.M., Merte, H., Jr., and Keller, R.B., "Low Velocity Subcooled Nucleate Flow Boiling at Various Orientations," ASME J. of Heat Trans., Vol. 117, 2, May, 1995, pp. 380386.

16. Ervin, J.S. and Merte, H., Jr., "A Fundamental Study of Nucleate Pool Boiling under Microgravity, " Final Report No. UMMEAM-91-08, The University of Michigan, Ann Arbor, Michigan, August 1991. Conducted under NASA Grant NAG3-663. 